been lacking, and it is relatively untested in community-living seniors who receive their meals in settings such as $\mathrm{CBN}$. We are also exploring mechanistic questions that relate to blood pressure control, such as the impact of the DASH diet on inflammation, which may lead to a better understanding of the underlying mechanism of action of the DASH diet. Our community partner, CBN, was awarded the DHHSACL nutrition innovation grant to conduct this 2-year study with CDN and RU-CCTS. The resulting study developed out of the community engaged pilot study represents a unique combination of community-centered care, within an implementation science framework (with embedded mechanistic measures under development). This is an example of the novel, full-spectrum approach to translational research that the RU-CCTS/CDN Community Engaged Research Core has been developing over the last decade. The research to characterize CBN clients' health status is now being extended to address cardiovascular health by way of intervening on diet quality and food insecurity, a key component of the social determinants of health, in partnership with agencies outside of the healthcare delivery system. The outcomes of the DASH Diet implementation study will also serve to inform the broader aging service provider network and the healthcare community about the impact of senior center congregate meal composition and services on health outcomes.

3205

A TL1 Team Approach to Examine Rural Tobacco Users' Barriers to Participating in Research

Rachel Elisabeth Damiani ${ }^{1}$, Neo Gebru, Robert Leeman and

Janice Krieger

${ }^{1}$ University of Florida Clinical and Translational Science Institute

OBJECTIVES/SPECIFIC AIMS: Our overarching theoretical framework is the health belief model (HBM). Guided by HBM, we aim to identify rural adults' perceived barriers and motivations to participating in research. Specifically, our research questions are listed below. RQ1: What socio-cultural factors influence rural tobacco users' intentions to a) participate in research studies and b) undertake tobacco cessation? RQ2: What bio-psychological and behavioral factors influence rural tobacco users' intentions to a) participate in research studies and b) undertake tobacco cessation? RQ3: How do rural tobacco users perceive citizen scientists as disseminators of a) tobacco cessation and b) recruitment messages? METHODS/ STUDY POPULATION: In Phase I of this multi-stage project, we are conducting in-depth interviews with approximately 30 tobacco users in rural Florida. The interview consists of semi-structured questions and multiple validated questionnaires. Specifically, we ask a series of questions about participants' barriers to participating in research. Additionally, we include questionnaires on participants' tobacco use history, nicotine dependence, motivation to quit, and willingness to participate in research studies. RESULTS/ANTICIPATED RESULTS: This study consists of two main phases. Data collection for Phase 1 of the study is ongoing, and we will discuss these recent findings. We anticipate data collection and data analysis to be finalized by May, 2019. Beginning in August 2019 through August 2020, we will focus on Phase II, which entails designing and implementing an intervention to increase rural tobacco users' willingness to participate in research. Given existing literature on other underrepresented groups in research, we anticipate that rural tobacco users will express that logistical barriers, such as transportation and time, prevent them from participating in research. Additionally, we anticipate these individuals may have socio-cultural barriers to participating in research, including distrust in the medical system and apprehension over discipline-specific terminology. DISCUSSION/SIGNIFICANCE OF IMPACT: The results of this formative research will be critical to our development of a targeted intervention to increase rural tobacco users' participation in research. Additionally, our interdisciplinary and community-based approach in this study acknowledges the importance of involving the target population in the research process, which is in line with NIH's updated model of translational research. We will discuss the process of collaborating with extension agents in rural counties in Florida to reach underrepresented communities.

3006

\section{Academic-Community Partnership and Capacity Strengthening for Deaf Community-engaged Research in the Dominican Republic}

Timothy De Ver Dye ${ }^{1}$, José Javier Sánchez², Pablo Taveras ${ }^{3}$, History Estill-Varner ${ }^{4}$, Wyatte Hall ${ }^{1}$, Alan Jesurum ${ }^{3}$, Shazia Siddiqi ${ }^{1}$, Joshua Mora ${ }^{1}$ and Zahira Quinones Tavarez ${ }^{1}$

${ }^{1}$ University of Rochester; ${ }^{2}$ Pontificia Universidad Católica Madre y Maestra; ${ }^{3}$ Asociación Nacional de Sordos de la Republica Dominicana and ${ }^{4}$ Discovering Deaf Worlds

OBJECTIVES/SPECIFIC AIMS: Deaf communities in many lowand middle-income countries (LMIC) struggle to organize, advocate, and reach social and health equity in their nations. In the Dominican Republic (DR), the health and social status of Deaf citizens is unclear, which obfuscates action and advocacy based on data. A set of successful pre-existing US-DR partnerships that function well but were not previously connected, organized around submission of a community-based NIH research grant and pilot work to support it. METHODS/STUDY POPULATION: Adapting the Partnership Synergy Framework for this purpose, we evaluate the partnership, its evolution, and its experience in implementing formative research. RESULTS/ANTICIPATED RESULTS: Our experience showed the local Deaf community organization easily recruited and interfaced with the Deaf community; presence of a trusted external organization facilitated entry of the PUCMM-UR research team; and stakeholders are enthusiastic about the partnership, its outputs, and the ability to recruit Dominican Deaf citizens into research. The partnership organized around production of an R21 to the Fogarty International Center (NIH), including Human Subjects certification, budget and scope of work negotiation, and inclusion of preliminary data. DISCUSSION/SIGNIFICANCE OF IMPACT: The engagement of Deaf communities globally is virtually non-existent in clinical and translational research. This partnership in the Dominican Republic shows that partners can organize around common goals and identify logistics required to produce pilot data and an NIH grant.

3125

Acceptability of a Narrative Video to Enhance the Use of Genetic Counseling in Latina Women at-risk of Hereditary Breast and Ovarian Cancer: Community Health Worker's Perspective

Alejandra Hurtado de Mendoza ${ }^{1}$, Kristi Graves, Sara Gómez-Trillos, Minna Song, Lyndsay Anderson, Claudia Campos, Pilar Carrera,

Nancy Ostrove, Paula Cupertino, Nathaly Gonzales and

Vanessa B. Sheppard

${ }^{1}$ Georgetown - Howard Universities

OBJECTIVES/SPECIFIC AIMS: The goal of the study was to assess the acceptability of a culturally targeted narrative video and identify 